\title{
4. TRIGONOMETRICAL PARALLAXES OF LB 3303 \\ AND LB 3459
}

\author{
J. CHURMS \\ Royal Observatory, Cape, South Africa \\ and
}

A. D. THACKER AY

Radcliffe Observatory, Pretoria, South Africa

\section{LB 3303}

Radcliffe spectra [1] of this star in 1961 confirmed Luyten's suspicion on the basis of colour and proper motion that this object is a bright white dwarf. The profile of $\mathrm{H} \gamma$ was found to be closely similar to that of $\mathrm{O}_{2}$ Eri B. Hill and Hill [2] have published photometry yielding $V=11.40$. The only known white dwarfs brighter than this are Sirius B, $\mathrm{o}_{2}$ Eri B, Procyon B, Feige 34 and CD $-38^{\circ} 10980$.

A trigonometrical parallax has been determined by one of us (J.C.) at the Royal Observatory, Cape. The result from 28 plates using 5 comparison stars is

Relative $\quad \pi_{t}=0.067 \pm 0.011$ (p.e.)

or following Binnendijk [3]

Absolute $\quad \pi_{t}=0.070 \pm 0.011$

The resulting absolute magnitude is

$$
M_{v}=+10.6 \pm 0.3
$$

This result is derived without the systematic correction of -0.002 to Cape parallaxes recommended by Jenkins [4] since Strand [5] has advised against such Yale-Jenkins system of corrections. (If the Yale-Jenkins precepts were followed the derived absolute parallax would become $+0.065 \pm 0.014$.)

The derived $M_{v}(+10.6)$ makes the star rather luminous for a white dwarf, but the observations are consistent with $M_{v}=+10.8$, the value for W 485 (with very similar colours) and the hotter object W 1346 (Eggen and Greenstein [6]). The star appears to be $0.8 \pm 0.3$ brighter than the Eggen-Greenstein [6] mean relation

$$
M_{v}=11.65+0.85(\mathrm{U}-\mathrm{V})
$$

Nevertheless, the parallax provides the final confirmation without doubt that the star is a relatively near white dwarf.

\section{LB 3459}

This is an extremely blue galactic star superposed on the Large Magellanic Cloud, discovered by Luyten on the basis of its proper motion. Radcliffe spectroscopy [7] confirmed its membership of the galactic foreground and also the variation in its 
spectrum already noted by Miss Cannon although only the first Radcliffe spectrum showed strong $\mathrm{HeI}$ lines, MgII 4481 and a peculiarly strong $\mathrm{Ca}^{+} \mathrm{K}$ line which cannot be interstellar. Spectroscopic monitoring has been continued and of 12 Radcliffe spectra taken between 1958 and 1968 all but the first still show weak helium lines.

The light also appears to be constant.

Although the spectrum does not suggest that of a white dwarf, the significant proper motion meant that a measurement of parallax was desirable and it was put on the Cape programme. The result from 27 plates using 5 comparison stars is given below, together with the Binnendijk correction from relative to absolute.

Relative $\quad \pi_{t}=-0.013 \pm 0.008$ (p.e.)

Absolute $\quad \pi_{t}=-0.010 \pm 0.008$

If we assume that the error of this determination is less than 4 times its probable error, we derive a minimum distance of 45 parsec, and with the Hills [2] photometry, $M_{v} \leq+7.8$. The star cannot be regarded as a white dwarf on the basis of its spectrum or absolute magnitude. On the other hand the fact that the star exhibits appreciable proper motion as discovered by Luyten sets an upper limit to the luminosity. A newly determined Cape proper motion of 0.038 , yields a tangential motion $T$ for various assumed distances as set out in Table I.

TABLE I

$\begin{array}{lccc}r \text { (parsec) } & M_{v} & T(\mathrm{~km} / \mathrm{s}) & z \text { (parsec) } \\ & \cdots & & \\ 45 & 7.8 & 8.3 & 24 \\ 180 & 4.8 & 33 & 96 \\ 360 & 3.3 & 66 & 192 \\ 720 & 1.8 & 132 & 384\end{array}$

With a small radial velocity (certainly less than $50 \mathrm{~km} / \mathrm{sec}$ ) we can say that

$$
+1.8<M_{v} \leqslant+7.8
$$

Thus LB 3459 appears to be an unusual object lying between the hot subdwarfs and the white dwarfs. He II 4686 appears weakly in absorption on some Radcliffe spectra and thus a revised classification 'OBp' is perhaps appropriate.

Table II summarises information about the two stars. Successive columns give (1) star designation from various catalogues, (2) galactic coordinates, (3) V, B - V, U- B from the Hills' photometry, (4) Spectral classification and derived $M_{v}$, (5) Trigonometrical parallax from this determination, (6) newly determined Cape proper motion (by J.C.), (7) previously published (CPC 50) proper motion.

\section{Acknowledgements}

We are indebted to Mrs. B. Brown, Mrs. M. C. Coetzee, and Miss A. J. Goedhals who performed the measures of the trigonometrical parallaxes at the Royal Observatory, Cape. 
TABLE II

\begin{tabular}{|c|c|c|c|c|c|c|c|c|}
\hline Star & $\begin{array}{l}l^{\mathrm{II}} \\
b^{\mathrm{II}}\end{array}$ & $\begin{array}{l}V \\
\mathrm{~B}-\mathrm{V} \\
\mathrm{U}-\mathrm{B}\end{array}$ & $\mathrm{Sp}$ & $\begin{array}{l}\pi_{t} \\
M_{i}\end{array}$ & (p.e.) & $\begin{array}{l}\text { Relative } \\
\mu \chi \\
\mu \delta\end{array}$ & (p.e.) & $\begin{array}{l}\text { CPC50 } \\
\mu \chi \\
\mu \delta\end{array}$ \\
\hline $\begin{array}{l}L B 3303 \\
-69^{\circ} 177 \\
\text { EG } 21\end{array}$ & $\begin{array}{r}286.2 \\
-43.7\end{array}$ & $\begin{array}{r}11.40 \\
+0.05 \\
-0.55\end{array}$ & DA & $\begin{array}{l}0^{\prime \prime} .070 \\
10.6\end{array}$ & $\begin{array}{l}(0.011) \\
(0.3)\end{array}$ & $\begin{array}{l}0^{\prime \prime} .038 \\
-0.104\end{array}$ & $\begin{array}{l}(0.004) \\
(0.004)\end{array}$ & \\
\hline $\begin{array}{c}L B 3459 \\
-69^{\circ} 389 \\
269696 \\
(\mathrm{HDE})\end{array}$ & $\begin{array}{r}280.5 \\
-32.2\end{array}$ & $\begin{array}{r}11.13 \\
-0.27 \\
-1.10\end{array}$ & $\begin{array}{l}\text { OBp } \\
\mathrm{p}\end{array}$ & $\begin{aligned} & -0^{\prime \prime} .010 \\
\leqslant & +7.8\end{aligned}$ & $(0.008)$ & $\begin{array}{l}-0^{\prime \prime} .008 \\
+0.038\end{array}$ & $\begin{array}{l}(0.004) \\
(0.004)\end{array}$ & $\begin{array}{l}-0.002(0.004) \\
+0.044(0.004)\end{array}$ \\
\hline
\end{tabular}

\section{References}

[1] Thackeray, A. D.: 1961, Monthly Notes Astron. Soc. S. Afr. 20, 40.

[2] Hill, P. W. and Hill, S. R.: 1966, Monthly Notices Roy. Astron. Soc. 133, 205.

[3] Binnendijk, L.: 1943, Bull. Astron. Inst. Neth. 10, 9.

[4] Jenkins, L. F.: 1952, General Catalogue of Trigonometric Stellar Parallaxes.

[5] Strand, K. Aa.: 1963, 'Basic Astronomical Data', Stars and Stellar Systems III, 55.

[6] Eggen, O. J. and Greenstein, J. L.: 1965, Astrophys. J. 141, 83.

[7] Feast, M. W., Thackeray, A. D., and Wesselink, A. J.: 1960, Monthly Notices Roy. Astron. Soc. 121, 383.

[8] Luyten, W. J.: 1959, A Search for Faint Blue Stars XVII, Minneapolis. 